\title{
Analysis of Double-Negative Materials with Surface Integral Equations and the Multilevel Fast Multipole Algorithm
}

\author{
Özgür Ergül ${ }^{1}$ and Levent Gürel ${ }^{2,3}$ \\ ${ }^{1}$ Department of Mathematics and Statistics, University of Strathclyde, G11XH, Glasgow, UK \\ ${ }^{2}$ Department of Electrical and Electronics Engineering, Bilkent University, TR-06800, Bilkent, Ankara, Turkey \\ ${ }^{3}$ Computational Electromagnetics Research Center (BiLCEM), Bilkent University, TR-06800, Bilkent, Ankara, Turkey \\ ozgur.ergul@strath.ac.uk, lgurel@bilkent.edu.tr
}

\begin{abstract}
We present a fast and accurate analysis of doublenegative materials (DNMs) with surface integral equations and the multilevel fast multipole algorithm (MLFMA). DNMs are commonly used as simplified models of metamaterials at resonance frequencies and are suitable to be formulated with surface integral equations. However, realistic metamaterials and their models are usually very large with respect to wavelength and their accurate solutions require fast algorithms, such as MLFMA. We consider iterative solutions of DNMs with MLFMA and we investigate the accuracy and efficiency of solutions when DNMs are formulated with two recently developed formulations, namely, the combined tangential formulation (CTF) and the electric and magnetic current combined-field integral equation (JMCFIE). Numerical results on canonical objects are consistent with previous results in the literature on ordinary objects.
\end{abstract}

\section{INTRODUCTION}

A popular approach for simulating metamaterials is homogenization. A metamaterial structure at a resonance frequency can be modeled as a homogeneous object with negative permittivity and permeability, i.e., as a double-negative material (DNM). Using the equivalence principle, a DNM can be formulated with the surface integral equations, which can be discretized and solved numerically. Recently, various surface formulations, such as the Poggio-Miller-Chang-HarringtonWu-Tsai (PMCHWT) formulation [1], the Müller formulation [2], and the electric and magnetic current combined-field integral equation (JMCFIE) [3], have been used to analyze DNMs [4],[5]. It has been shown that homogenization can provide fast analysis of metamaterial structures before their detailed analysis via full-wave solvers.

Homogenization of metamaterials is relatively easy; but, efficient solutions of the resulting DNMs may not be. Although surface integral equations require only the discretization of boundaries, the resulting matrix equations can be very large because realistic metamaterials are usually large with respect to wavelength. Hence, fast and efficient methods, such as the multilevel fast multipole algorithm (MLFMA) [6], are required for the solution of large metamaterial problems, even when they are homogenized. Applying MLFMA to homogeneous materials, including DNMs, is quite straightforward, but the number of iterations must be small for efficient solutions, and thus the choice of the surface formulation is critical for efficient solutions.

In this study, we present iterative solutions of DNMs using MLFMA. Problems are formulated with two recently developed formulations, namely, the combined tangential formulation (CTF) [7] and JMCFIE [3], and discretized with the Rao-Wilton-Glisson (RWG) functions [8]. Accuracy and efficiency of the numerical solutions are investigated on canonical problems involving the sphere geometry. We show that the conventional JMCFIE (with $\alpha=0.5$ combination parameter) provides efficient solutions but relatively inaccurate results. In addition, accuracy of the simulations can be significantly improved using CTF, instead of JMCFIE. These observations are consistent with earlier results obtained for ordinary materials [9]. We also show that the combination parameter of JMCFIE can be increased towards unity to improve the accuracy of JMCFIE, without sacrificing the efficiency.

\section{Numerical Solutions of Surface Formulations}

For homogeneous objects, discretizations of surface formulations lead to $2 N \times 2 N$ dense matrix equations in the form of

$$
\left[\begin{array}{ll}
\overline{\boldsymbol{Z}}^{(11)} & \overline{\boldsymbol{Z}}^{(12)} \\
\overline{\boldsymbol{Z}}^{(21)} & \overline{\boldsymbol{Z}}^{(22)}
\end{array}\right] \cdot\left[\begin{array}{l}
\boldsymbol{x} \\
\boldsymbol{y}
\end{array}\right]=\left[\begin{array}{l}
\boldsymbol{v}^{(1)} \\
\boldsymbol{v}^{(2)}
\end{array}\right]
$$

Using JMCFIE and a Galerkin discretization, $\overline{\boldsymbol{Z}}^{(11)}=\overline{\boldsymbol{Z}}^{(22)}$ and

$$
\begin{aligned}
Z_{m n}^{(11)} & =\alpha \int_{S_{m}} d \boldsymbol{r} \boldsymbol{t}_{m}(\boldsymbol{r}) \cdot\left(\mathcal{T}_{o}+\mathcal{T}_{i}\right)\left\{\boldsymbol{b}_{n}\right\}(\boldsymbol{r}) \\
& +(1-\alpha) \int_{S_{m}} d \boldsymbol{r} \boldsymbol{t}_{m}(\boldsymbol{r}) \cdot \hat{\boldsymbol{n}} \times\left(\mathcal{K}_{o}-\mathcal{K}_{i}\right)\left\{\boldsymbol{b}_{n}\right\}(\boldsymbol{r}) \\
& -(1-\alpha) \int_{S_{m}} d \boldsymbol{r} \boldsymbol{t}_{m}(\boldsymbol{r}) \cdot \boldsymbol{b}_{n}(\boldsymbol{r})
\end{aligned}
$$




$$
\begin{aligned}
Z_{m n}^{(12)} & =(1-\alpha) \int_{S_{m}} d \boldsymbol{r} \boldsymbol{t}_{m}(\boldsymbol{r}) \cdot \hat{\boldsymbol{n}} \times\left(\eta_{o}^{-1} \mathcal{T}_{o}-\eta_{i}^{-1} \mathcal{T}_{i}\right)\left\{\boldsymbol{b}_{n}\right\}(\boldsymbol{r}) \\
& -\alpha \int_{S_{m}} d \boldsymbol{r} \boldsymbol{t}_{m}(\boldsymbol{r}) \cdot\left(\eta_{o}^{-1} \mathcal{K}_{o}+\eta_{i}^{-1} \mathcal{K}_{i}\right)\left\{\boldsymbol{b}_{n}\right\}(\boldsymbol{r}) \\
& -\frac{1}{2} \alpha\left(\eta_{o}^{-1}-\eta_{i}^{-1}\right) \int_{S_{m}} d \boldsymbol{r} \boldsymbol{t}_{m}(\boldsymbol{r}) \cdot \hat{\boldsymbol{n}} \times \boldsymbol{b}_{n}(\boldsymbol{r}) \\
Z_{m n}^{(21)} & =-(1-\alpha) \int_{S_{m}} d \boldsymbol{r} \boldsymbol{t}_{m}(\boldsymbol{r}) \cdot \hat{\boldsymbol{n}} \times\left(\eta_{o} \mathcal{T}_{o}-\eta_{i} \mathcal{T}_{i}\right)\left\{\boldsymbol{b}_{n}\right\}(\boldsymbol{r}) \\
& +\alpha \int_{S_{m}} d \boldsymbol{r} \boldsymbol{t}_{m}(\boldsymbol{r}) \cdot\left(\eta_{o} \mathcal{K}_{o}+\eta_{i} \mathcal{K}_{i}\right)\left\{\boldsymbol{b}_{n}\right\}(\boldsymbol{r}) \\
& +\frac{1}{2} \alpha\left(\eta_{o}-\eta_{i}\right) \int_{S_{m}} d \boldsymbol{r} \boldsymbol{t}_{m}(\boldsymbol{r}) \cdot \hat{\boldsymbol{n}} \times \boldsymbol{b}_{n}(\boldsymbol{r})
\end{aligned}
$$

In (2)-(4), $\boldsymbol{t}_{m}$ and $\boldsymbol{b}_{n}$ for $m, n=1,2, \ldots, N$ represent the testing and basis functions with spatial supports of $S_{m}$ and $S_{n}$, respectively, $\alpha \in[0,1]$ is the combination parameter, $\hat{\boldsymbol{n}}$ is the unit normal vector at the observation point $\boldsymbol{r}$, and $\eta_{u}=$ $\sqrt{\mu_{u}} / \sqrt{\epsilon_{u}}$ is the intrinsic impedance of the outer $(u=o)$ and inner $(u=i)$ media. The integro-differential operators are defined as

$$
\begin{aligned}
\mathcal{T}_{u}\left\{\boldsymbol{b}_{n}\right\}(\boldsymbol{r}) & =i k_{u} \int_{S_{n}} d \boldsymbol{r}^{\prime} \boldsymbol{b}_{n}\left(\boldsymbol{r}^{\prime}\right) g_{u}\left(\boldsymbol{r}, \boldsymbol{r}^{\prime}\right) \\
& +\frac{i}{k_{u}} \int_{S_{n}} d \boldsymbol{r}^{\prime} \nabla^{\prime} \cdot \boldsymbol{b}_{n}\left(\boldsymbol{r}^{\prime}\right) \nabla g_{u}\left(\boldsymbol{r}, \boldsymbol{r}^{\prime}\right) \\
\mathcal{K}_{u}\left\{\boldsymbol{b}_{n}\right\}(\boldsymbol{r}) & =\int_{\mathrm{PV}, S_{n}} d \boldsymbol{r}^{\prime} \boldsymbol{b}_{n}\left(\boldsymbol{r}^{\prime}\right) \times \nabla^{\prime} g_{u}\left(\boldsymbol{r}, \boldsymbol{r}^{\prime}\right),
\end{aligned}
$$

where PV indicates the principal value of the integral, $k_{u}=$ $\omega \sqrt{\epsilon_{u}} \sqrt{\mu_{u}}$ is the wavenumber, and

$$
g_{u}\left(\boldsymbol{r}, \boldsymbol{r}^{\prime}\right)=\frac{\exp \left(i k_{u}\left|\boldsymbol{r}-\boldsymbol{r}^{\prime}\right|\right)}{4 \pi\left|\boldsymbol{r}-\boldsymbol{r}^{\prime}\right|}
$$

denotes the homogeneous-space Green's function in the phasor domain using the $e^{-i \omega t}$ time dependence. The elements of the right-hand-side vectors in (1) are derived as

$$
\begin{aligned}
v_{m}^{(1)} & =-(1-\alpha) \int_{S_{m}} d \boldsymbol{r} \boldsymbol{t}_{m}(\boldsymbol{r}) \cdot \hat{\boldsymbol{n}} \times \boldsymbol{H}^{i n c}(\boldsymbol{r}) \\
& -\alpha \eta_{o}^{-1} \int_{S_{m}} d \boldsymbol{r} \boldsymbol{t}_{m}(\boldsymbol{r}) \cdot \boldsymbol{E}^{i n c}(\boldsymbol{r}) \\
v_{m}^{(2)} & =(1-\alpha) \int_{S_{m}} d \boldsymbol{r} \boldsymbol{t}_{m}(\boldsymbol{r}) \cdot \hat{\boldsymbol{n}} \times \boldsymbol{E}^{i n c}(\boldsymbol{r}) \\
& -\alpha \eta_{o} \int_{S_{m}} d \boldsymbol{r} \boldsymbol{t}_{m}(\boldsymbol{r}) \cdot \boldsymbol{H}^{i n c}(\boldsymbol{r}),
\end{aligned}
$$

where $\boldsymbol{E}^{i n c}$ and $\boldsymbol{H}^{i n c}$ represent the incident electric and magnetic fields created by external sources.

JMCFIE is a mixed formulation involving directly and rotationally tested electromagnetic fields. Using a Galerkin discretization, e.g., using the same set of the RWG functions as the basis and testing functions, JMCFIE involves well-tested

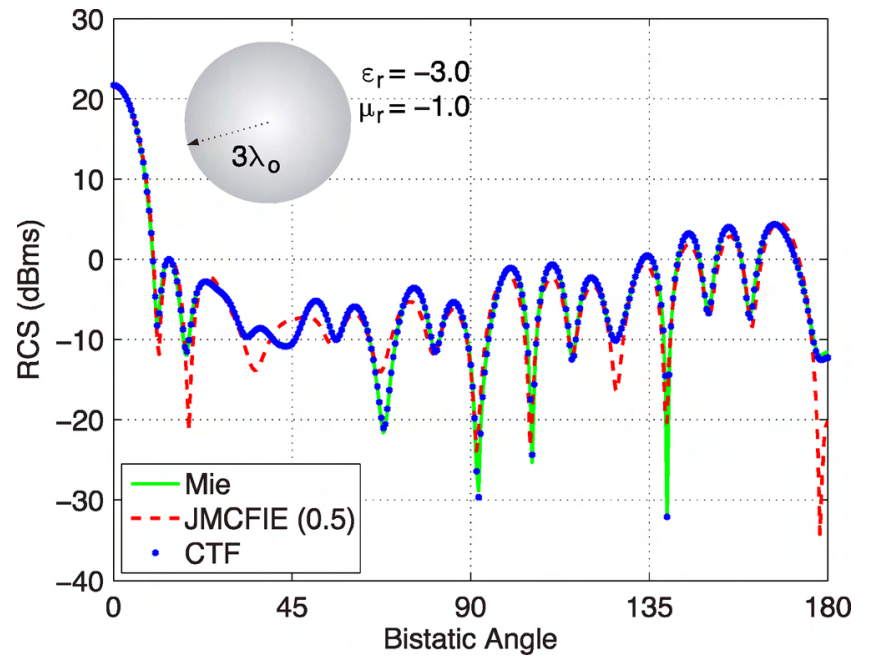

Fig. 1. Solutions of a scattering problem involving a sphere of radius $0.3 \mathrm{~m}$ at $3 \mathrm{GHz}$, i.e., when the radius of the sphere is approximately $3 \lambda_{o}$. The relative permittivity and permeability of the sphere are -3.0 and -1.0 , respectively. The bistatic RCS is plotted from $0^{\circ}$ to $180^{\circ}$, where $0^{\circ}$ corresponds to the forward-scattering direction.

identity operators, i.e.,

$$
\begin{aligned}
\overline{\boldsymbol{I}}_{m n} & =\int_{S_{m}} d \boldsymbol{r} \boldsymbol{t}_{m}(\boldsymbol{r}) \cdot \boldsymbol{b}_{n}(\boldsymbol{r}) \\
& =\int_{S_{m}} d \boldsymbol{r} \boldsymbol{t}_{m}(\boldsymbol{r}) \cdot \int_{S_{n}} \delta\left(\boldsymbol{r}, \boldsymbol{r}^{\prime}\right) \boldsymbol{b}_{n}\left(\boldsymbol{r}^{\prime}\right),
\end{aligned}
$$

which is a major error source for low-order discretizations [10]. CTF can be seen as a special case of JMCFIE and it is obtained by setting $\alpha=1$ in (2)-(4), (8), and (9). Note that well-tested identity operators disappear in CTF; this explains why it is more accurate than JMCFIE.

\section{NUMERICAL RESULTS}

In order to test the accuracy and efficiency of the solutions of DNMs, we consider scattering problems involving the sphere geometry. All objects are located in free space and illuminated by plane waves. Problems are formulated with CTF and JMCFIE and discretized with the RWG functions on $\lambda_{o} / 10$ triangles, where $\lambda_{o}$ is the wavelength in the host medium (free space). Both near-field and far-field interactions are calculated with maximum $1 \%$ error. Solutions are performed using the biconjugate-gradient-stabilized (BiCGStab) algorithm [11] accelerated with MLFMA. Iterative convergences are also accelerated with the four-partition blockdiagonal preconditioner (4PBDP) [9] for the conventional JMCFIE $(\alpha=0.5)$. Iterations are carried out until the residual error is reduced to below 0.001 .

Fig. 1 presents the solution of a scattering problem involving a sphere of radius $0.3 \mathrm{~m}$ at $3 \mathrm{GHz}$. The relative permittivity and permeability of the sphere are -3.0 and -1.0 , respectively. For numerical solutions, the problem is discretized with 65,724 unknowns. Fig. 1 depicts the radar cross section (RCS) on the E-plane as a function of the bistatic observation angle from $0^{\circ}$ to $180^{\circ}$, where $0^{\circ}$ corresponds to the forward-scattering 

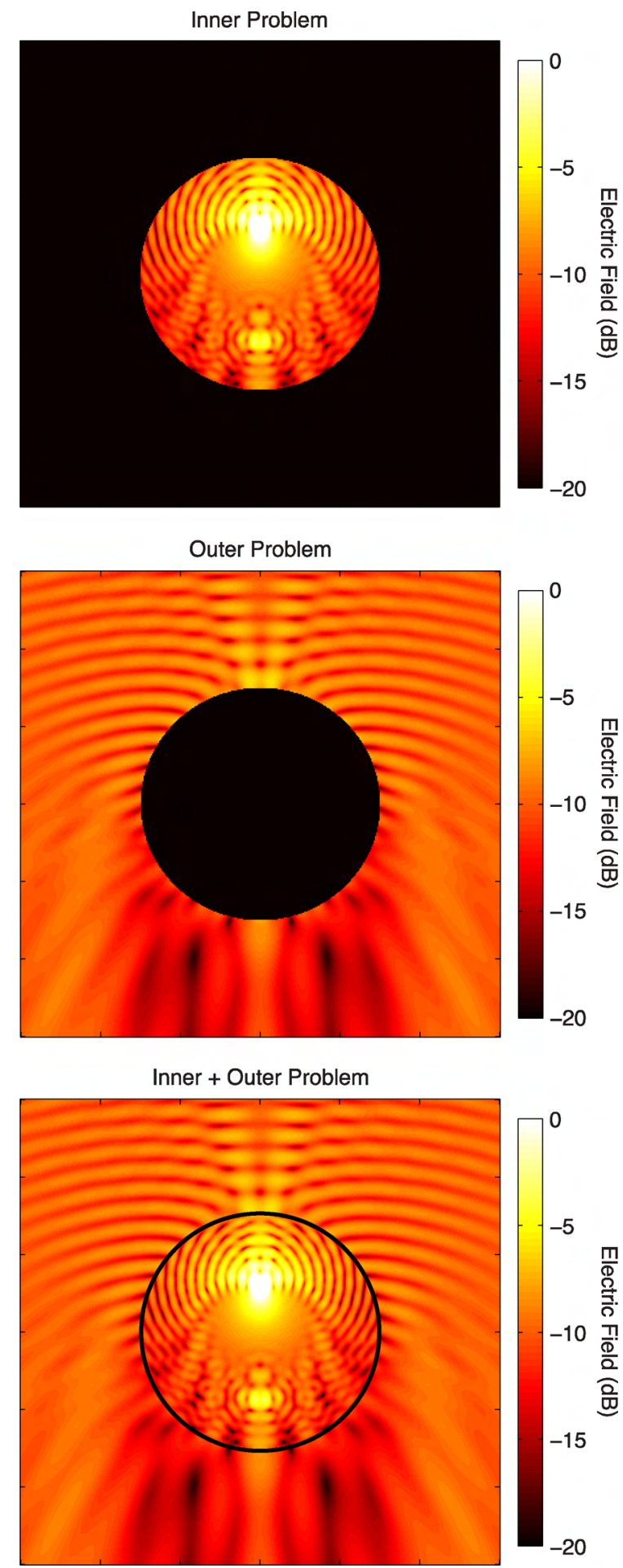

Fig. 2. Solution of a scattering problem involving a sphere of radius $0.3 \mathrm{~m}$ at $3 \mathrm{GHz}$, i.e., when the radius of the sphere is approximately $3 \lambda_{o}$. Both the relative permittivity and permeability of the sphere are -2.0 . The sphere is illuminated by a plane wave from the top. The total electric field in the vicinity of the sphere is plotted for the inner and outer problems, in addition to the complete plot obtained via their superposition.

direction. Computational values obtained with $\mathrm{CTF}$ and the conventional JMCFIE $(\alpha=0.5)$ are compared to the analytical Mie-series results. It can be observed that CTF results agree very well with the analytical results. However, the same level of accuracy is not provided with JMCFIE. In Fig. 1, this is clearly visible at various observation angles, e.g., in the backscattering direction $\left(180^{\circ}\right)$.

Fig. 2 presents the solution of another scattering problem involving a sphere of radius $0.3 \mathrm{~m}$ at $3 \mathrm{GHz}$. In this case, both the relative permittivity and permeability of the sphere are selected as -2.0 . The sphere is illuminated by a plane wave from the top and the problem is formulated with CTF. Fig. 2 depicts the total electric field in the vicinity of the sphere on the E-plane for the inner and outer problems. The maximum electric field value is normalized to $0 \mathrm{~dB}$. For the inner/outer problem, the equivalent currents provided by MLFMA are allowed to radiate into a homogeneous space with the electrical parameters of the inner/outer medium assumed everywhere. Hence, for the inner/outer problem, any radiation outside/inside the sphere can be interpreted as numerical error. It can be observed that these unwanted radiations are below $-20 \mathrm{~dB}$, verifying the accuracy of the solution. As also depicted in Fig. 2, the complete plot of the electric field can be obtained by superimposing the plots for the inner and outer problems. It is remarkable that a focusing is observed in the upper part of the sphere as a result of the negative refractive index of the object.

For a more quantitative comparison of the formulations, we present the solution of larger scattering problems involving a sphere of radius $0.3 \mathrm{~m}$ at $6 \mathrm{GHz}$. The sphere is illuminated by a plane wave and discretized with 264,006 unknowns. Different values for the relative permittivity and permeability are considered. Fig. 3 depicts the relative error in the far-zone electric field obtained with different formulations. In addition to CTF and the conventional JMCFIE ( $\alpha=0.5$ ), we consider JMCFIE with $\alpha=0.9$. To find the relative error, the copolar electric field in the far-zone on the E-plane is sampled at $\pi / 360$ intervals, i.e., we compute

$$
f[n]=\lim _{r \rightarrow \infty}\left\{r E_{\varphi}(r, \varphi[n])\right\}, \quad \varphi[n]=(n-1) \pi / 360
$$

for $n=1,2, \ldots, 361$. Then, the error is defined as

$$
\Delta=\frac{\left\|\boldsymbol{f}_{C}-\boldsymbol{f}_{A}\right\|}{\left\|\boldsymbol{f}_{A}\right\|},
$$

where $\|\cdot\|$ represents the 2-norm and $\boldsymbol{f}_{C}$ and $\boldsymbol{f}_{A}$ are vectors of 361 elements containing the computational and analytical values, respectively. Fig. 3 shows that the relative error for the conventional JMCFIE $(\alpha=0.5)$ is generally higher than $1 \%$, which may not be acceptable considering that the matrix elements are computed with maximum $1 \%$ error. As also shown in Fig. 3, the accuracy is significantly improved by using CTF or increasing the amount of CTF in JMCFIE, i.e., using JMCFIE with $\alpha=0.9$, instead of the conventional JMCFIE.

As complementary data, Fig. 4 depicts the number of BiCGStab iterations for the solution of the scattering problems shown in Fig. 3. Iterative solutions of the conventional JMCFIE $(\alpha=0.5)$ are accelerated with 4PBDP; but this preconditioner is not useful for CTF and JMCFIE with $\alpha=$ 


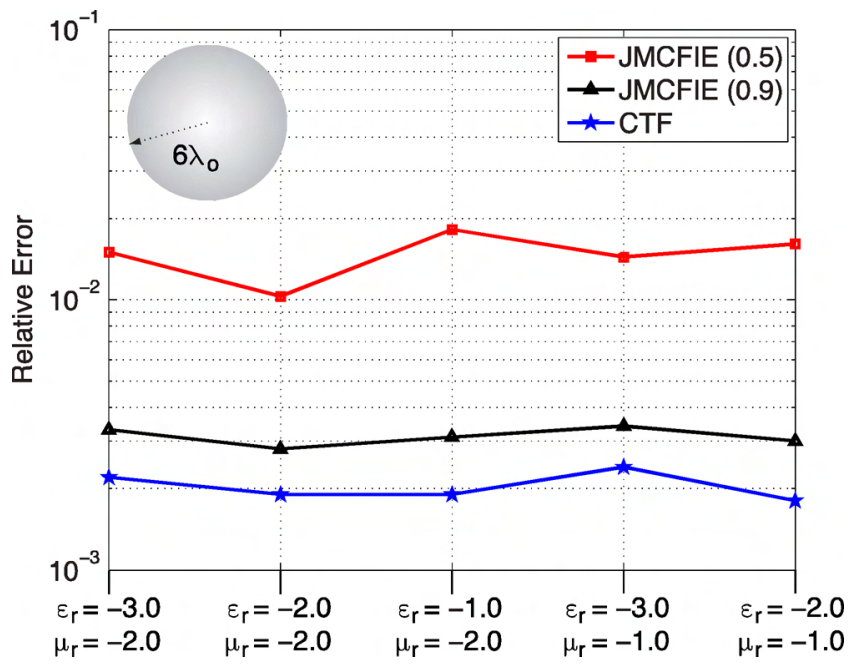

Fig. 3. The relative error in numerical solutions of scattering problems involving a sphere of radius $0.3 \mathrm{~m}$ at $6 \mathrm{GHz}$, i.e., when the radius of the sphere is approximately $6 \lambda_{o}$. Errors in the computational values of the far-zone electric field with respect to the analytical solution are plotted for different formulations and material properties.

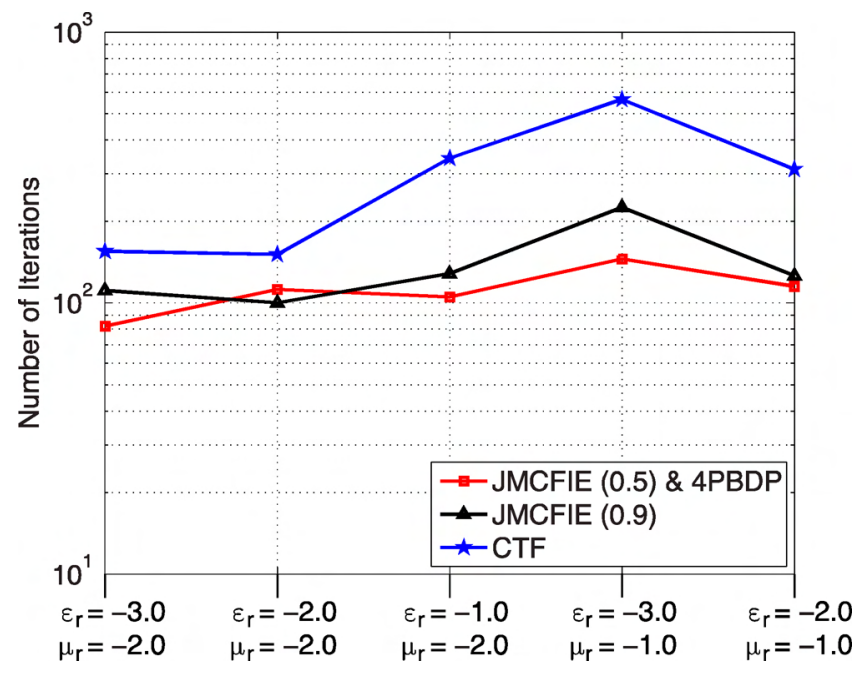

Fig. 4. The number of BiCGStab iterations (for $10^{-3}$ residual error) required in numerical solutions of scattering problems involving a sphere of radius $0.3 \mathrm{~m}$ at $6 \mathrm{GHz}$, i.e., when the radius of the sphere is approximately $6 \lambda_{o}$.

0.9. It can be observed that JMCFIE (both with $\alpha=0.5$ and with $\alpha=0.9$ ) provides very efficient solutions, compared to CTF. Considering the results in Figs. 3 and 4, JMCFIE with $\alpha=0.9$ seems a good choice for both efficient and accurate solutions.

\section{CONCLUSIONS}

This paper presents the analysis of DNMs with surface integral equations and MLFMA. To the best of the authors' knowledge, MLFMA is applied to DNMs for the first time here. Numerical results obtained with conventional formulations are in agreement with previous results obtained for ordinary materials. Specifically,

- the conventional JMCFIE with $\alpha=0.5$ provides more efficient solutions but less accurate results,
- CTF provides less efficient solutions but more accurate results, and

- the combination parameter of JMCFIE provides a tradeoff between accuracy and efficiency.

Numerical experiments on canonical objects show that JMCFIE with $\alpha=0.9$ is a good choice for efficient and accurate solutions.

\section{ACKNOWLEDGMENT}

This work was supported by the Scientific and Technical Research Council of Turkey (TUBITAK) under Research Grant 110E268, by the Centre for Numerical Algorithms and Intelligent Software (EPSRC-EP/G036136/1), and by contracts from ASELSAN and SSM.

\section{REFERENCES}

[1] A. J. Poggio and E. K. Miller, "Integral equation solutions of threedimensional scattering problems," in Computer Techniques for Electromagnetics, R. Mittra, Ed. Oxford: Pergamon Press, 1973, Chap. 4.

[2] C. Müller, Foundations of the Mathematical Theory of Electromagnetic Waves. New York: Springer, 1969.

[3] P. Ylä-Oijala and M. Taskinen, "Application of combined field integral equation for electromagnetic scattering by dielectric and composite objects," IEEE Trans. Antennas Propagat., vol. 53, no. 3, pp. 11681173, Mar. 2005.

[4] Y. A. Liu and W. C. Chew, "Stability of surface integral equation for left-handed materials," IET Microw. Antennas Propag., vol. 1, no. 1, pp. 84-89, Mar. 2007.

[5] J. Rivero, J. M. Taboada, L. Landesa, F. Obelleiro, and I. Garcia-Tunon, "Surface integral equation formulation for the analysis of left-handed metamaterials," Opt. Express, vol. 18, no. 15, pp. 15876-15886, Jul. 2010.

[6] J. Song, C.-C. Lu, and W. C. Chew, "Multilevel fast multipole algorithm for electromagnetic scattering by large complex objects," IEEE Trans. Antennas Propag., vol. 45, no. 10, pp. 1488-1493, Oct. 1997.

[7] P. Ylä-Oijala, M. Taskinen, and S. Järvenpää, "Surface integral equation formulations for solving electromagnetic scattering problems with iterative methods," Radio Sci., vol. 40, RS6002, doi:10.1029/2004RS003169, Nov. 2005.

[8] S. M. Rao, D. R. Wilton, and A. W. Glisson, "Electromagnetic scattering by surfaces of arbitrary shape," IEEE Trans. Antennas Propag., vol. 30, no. 3, pp. 409-418, May 1982.

[9] Ö. Ergül and L. Gürel, "Comparison of integral-equation formulations for the fast and accurate solution of scattering problems involving dielectric objects with the multilevel fast multipole algorithm," IEEE Trans. Antennas Propag., vol. 57, no. 1, pp. 176-187, Jan. 2009.

[10] Ö. Ergül and L. Gürel, "Discretization error due to the identity operator in surface integral equations," Comput. Phys. Comm., vol. 180, no. 10, pp. 1746-1752, Oct. 2009.

[11] H. van der Vorst, "Bi-CGSTAB: A fast and smoothly converging variant of Bi-CG for the solution of nonsymmetric linear systems," SIAM J. Sci. Stat. Comput., vol. 13, no. 2, pp. 631-644, Mar. 1992. 